\title{
Hyperspectral remote sensing for assessment of chlorophyll sufficiency levels in mature oil palm (Elaeis guineensis) based on frond numbers: analysis of decision tree and random forest
}

\begin{abstract}
A common practice of chlorophyll (chl) determination has been using the chemical analysis that is destructive and time-consuming. A current prospective alternative method such hyperspectral remote sensing offers a non-destructive measurement of chl which provides a result in the rapid and real-time manner. Therefore, the ultimate aims of this study were to propose the chls (chl a, chl b, total chl content (TCC) and relative chl content (RCC)) sufficiency levels via Jenks Natural Breaks (JNB) classification and recommend the best subset of chl-sensitive wavelengths, frond number and classifier for classifying the chls according to the designated sufficiency levels via hyperspectral remote sensing platform. In order to achieve the objectives, an experiment was conducted on mature Tenera palm stands (12 and 15 years old) with $\mathrm{N}$ treatments varied from 0 to $2 \mathrm{~kg}$. The Jenks Natural Breaks (JNB) classification was proposed to determine the chl sufficiency levels. Feature selection was carried out to select the chl-sensitive wavelengths while Decision Tree (DT) and Random Forest (RF) classifiers were used to classify the chl sufficiency levels using the selected wavelengths. Results from this study showed that the chl-sensitive wavelengths mostly belong to the red-edge region and importantly, they were frond age-dependent. Generally, the classification accuracy tended to decrease as frond gets older. The RF performed the best in discriminating the chl sufficiency levels with accuracy ranging from 92.79 to $98.77 \%$ compared to $56.30-90.48 \%$ achieved by DT. In the nutshell, the execution of frond 9 was more ideal than frond 17 for monitoring chl content of mature oil palm via remote sensing and RF portrayed promising insight in assessing chls content of mature oil palm.
\end{abstract}

Keyword: Frond age; Hyperspectral; Machine learning; Jenks Natural Break classification 